\title{
Más es más. Una relectura "Venturiana" de la arquitectura española de los siglos XV y XVI a partir del Palacio Avellaneda
}

Fernando Luis Martínez Nespral

Instituto de Arte Americano e Investigaciones Estéticas "Mario J. Buschiazzo", Facultad de Arquitectura, Diseño y Urbanismo, Universidad de Buenos Aires, Argentina

fmnespral@gmail.com

\section{Resumen}

La arquitectura del denominado "primer renacimiento español" se caracteriza por la combinación de elementos de origen medieval (ya sea del gótico como del mudéjar) con las nuevas influencias del renacimiento italiano. Hasta aquí reseñamos un tema conocido, pero casualmente, a partir de dos recientes visitas a un ejemplo excepcional, el Palacio Avellaneda, nos han surgido una serie de reflexiones acerca del período y de su tratamiento en la historiografía que proponemos compartir. Se ha interpretado tradicionalmente a estas obras como fenómenos donde huellas del pasado medieval se proyectan en un presente signado por la novedad renacentista. Entendemos, por el contrario que los elementos mudéjares no conforman una huella o sombra de un fenómeno pasado sino una componente presente en la cultura española cuyos rasgos, lejos de ser accesorios o superficiales, definen la estructura a partir de la cual dichos edificios han sido concebidos. El Palacio Avellaneda refleja esta problemática de manera sorprendentemente clara comunicando la complejidad y contradicción de una sociedad bifronte. Comunicación, complejidad, contradicción. Salta a la vista que esta aproximación no sería posible sin el aporte del recientemente fallecido Robert Venturi, proponemos pues una relectura en clave "Venturiana" de la obra y su período.

Palabras clave: Arquitectura, Primer Renacimiento, Palacio Avellaneda. 


\title{
More is more. A "Venturian" rereading of 15th and 16th centuries Spanish architecture from the Avellaneda Palace
}

\begin{abstract}
The architecture of the so-called "first Spanish Renaissance" is characterized by the combination of elements of medieval origin (either Gothic or Mudejar) with the new influences of the Italian Renaissance. Until here, we have mentioned a well-known topic, but coincidentally, from two recent visits to an outstanding example, the Avellaneda Palace, emerged a series of reflections about the period and its treatment in the historiography that we propose to share. These works have traditionally been interpreted as phenomena where traces of the medieval past are projected into a present marked by Renaissance. On the contrary, we understand that the Mudejar elements are not a trace or shadow of a past phenomenon, but rather a component present in Spanish culture whose features, far from being accessory or superficial, define the structure from which these buildings have been conceived. Avellaneda Palace reflects this problem in a surprisingly clear way, communicating the complexity and contradiction of a two-faced society. Communication, complexity, contradiction. It is obvious that this approach would not be possible without the contribution of the recently deceased Robert Venturi, so we propose a "Venturian" rereading of the work and its period.
\end{abstract}

Keywords: Architecture, First Renaissance, Avellaneda Palace.

\section{El viejo juego de moros y cristianos, una encrucijada epistemológica}

"...el prejuicio es la parte inconsciente de la ideología de una sociedad." (Todorov 2007 [1989] 403).

Comenzamos por "Nosotros y los otros" de Todorov porque entendemos que la cultura española desde la Edad Moderna, está definida a partir de su identificación exclusiva con su componente cristiana y la consiguiente diferenciación y distanciamiento de su componente islámica.

Todos los hechos históricos contradicen esta interpretación, desde la demografía hasta la genealogía. Sabemos a ciencia cierta que no existió una "invasión" de "moros" (sería mejor decir musulmanes del norte de África) ni una expulsión de "moriscos" (seria también más apropiado decir descendientes de musulmanes ibéricos) con un volumen suficiente como para generar un impacto demográfico significativo. 
Por el contrario, sabemos que lo que realmente sucedió, fue que el mismo sustrato poblacional ibérico, dominado y convertido a diversas religiones por los distintos conquistadores de turno (romanos y visigodos entre ellos) acogió primero la dominación de los musulmanes norafricanos y luego la de los reinos cristianos del norte de la Península convirtiéndose a la religión de los que circunstancialmente detentaban el poder como había sucedido anteriormente.

Por otra parte, sabemos también que, la llamada "Edad Media”, un periodo caracterizado por la atomización e inestabilidad política en toda Europa, estuvo definida en la Península ibérica por múltiples alianzas cruzadas entre sus habitantes de diversas religiones, ya sea cristianos, como judíos y musulmanes.

Estas alianzas incluyeron en todos los estratos de la sociedad los consiguientes vínculos matrimoniales por lo cual, más allá de los argumentos demográficos expresados precedentemente la existencia de habitantes descendientes "puros" con antepasados de una sola religión tendría un impacto demográfico y culturalmente intrascendente.

Por lo tanto, los argumentos que aun hoy se siguen repitiendo, incluso en ámbitos académicos, acerca de la clara diferenciación entre moros y cristianos y de una guerra de "Reconquista" por la cual los segundos recuperaron el territorio perdido por sus ancestros frente a los primeros son a la vez falaces y racistas. ${ }^{1}$

Sin embargo, vemos como la historiografía se esmera en señalar esta falsa diferencia y asignarle una identidad particular, definida y opuesta para quienes profesaban una u otra religión, y por ende a su producción arquitectónica que es lo que nos ocupa.

1 Existe una copiosa bibliografía respecto al mito de la "Reconquista" y otros ligados a la relación entre cristianos y musulmanes en la Edad Media, como: "La invención de España" de Henry Kamen, Espasa 2020 y "Cuando fuimos árabes" de Emilio González Ferrín, Almuzara, 2018, por mencionar algunos de los más recientes. Pero ideas similares vienen siendo sostenidas desde hace casi un siglo como en el caso del libro "La invención de España" (homónimo al precedente) de Inman Fox, Cátedra, 1997, el ya clásico "España en su historia, cristianos, moros y judíos", Losada, 1948 de Américo Castro, estando la idea incluso presente en la "España invertebrada" de Ortega y Gasset, Espasa Calpe, 1921. De hecho, la superabundancia de textos al respecto que se repiten década a década, se convierte en una demostración de la sorprendente vigencia de estos mitos en la construcción de la identidad española, al punto que subsisten en el imaginario popular, y ocasionalmente en discursos pseudo-eruditos, pese a un siglo de debates que demostraron lo contrario. 
Las obras dan testimonio en sentido contrario, en todo el territorio peninsular abundan edificios destinados a cristianos donde se pueden encontrar elementos de origen indudablemente islámico y para definir esta realidad compleja y contradictoria (Venturi, 1968 [1966]), José Amador de los Ríos hubo de crear la categoría también racista de "Mudéjar"2 que define a la arquitectura a partir de la filiación religiosa y supuestamente étnica de sus autores, musulmanes ibéricos que continuaban habitando los territorios conquistados por cristianos.

Pero, y basados en lo expresado anteriormente, esta condición mestiza (mejor sería decir híbrida) donde coexisten elementos de origen musulmán y cristiano, sería aplicable a todos los peninsulares, por lo cual, entendido así, llegaríamos al absurdo por el cual todas las expresiones arquitectónicas ibéricas serían pasibles de ser denominadas mudéjares.

Hasta en las arquitecturas de Asturias "la indómita" o en los Pirineos se encuentran rasgos islámicos y, por el contrario, hasta en la Córdoba califal existe lo opuesto, comenzando por el arco de herradura, de probado origen visigótico.

Por ello, en un contexto definido fundamentalmente por la interculturalidad como fenómeno dominante, no tiene sentido explicar a sus productos partiendo de dos hipotéticos modelos "puros" que son justamente los casos más raros o incluso ausentes.

Por lo tanto, desde nuestra lectura, es la misma necesidad de ver como entidades separadas y diferentes a los rasgos del arte islámico y cristiano en la Península la que provoca serios errores de interpretación.

Un texto clásico que explica el periodo que nos ocupa se denomina "Proyecciones del Arte Islámico en la Arquitectura de nuestro primer Renacimiento” de Miguel Ángel Castillo Oreja (Castillo Oreja, 1985).

Partiendo desde el título, en esta concepción, lo islámico, lejos de ser visto en su hibridez, no solo se presenta como un rasgo diferenciado e identificable, sino que se le asigna un carácter pasado, se lo entiende como expresión de un proceso histórico concluido que se "proyecta" en el presente definido por otras pautas.

2 Ver: "El estilo mudéjar en la arquitectura" discurso de José Amador de los Ríos: leído en junta pública de 19 de junio de 1859 [Real Academia de Bellas Artes de San Fernando, Madrid]. 
Merece destacarse en este sentido el uso de adjetivos calificativos para definir a la componente islámica, mencionamos recién "proyección”, pero también se han usado "huellas" o hasta "sombra"3, todas ellas rastros visibles de un objeto distante, incluso en el caso de "sombras" con evidentes connotaciones negativas.

Contrariamente a la distancia impuesta a lo islámico, el texto de Castillo Oreja define al Renacimiento con la cercanía de lo "nuestro" y a la vez como aquello vigente y presente pues es el soporte sobre el cual el pasado islámico se proyecta.

Esta interpretación, también evidentemente racista, asume una supuesta "hispanidad" Occidental y cristiana para la cual el Renacimiento es algo propio y actual (aunque se trate en realidad para el momento de una distante moda italiana cuyos efectos recién comenzaban a advertirse) mientras que lo islámico es necesariamente un rasgo ajeno y pasado que, aun cuando resulta evidente, solo puede ser interpretado como una proyección de tiempos ya pretéritos.

Todorov explica este proceso claramente como requisito de la exotización: “...es preciso bloquear el proceso habitual de asimilación (del otro) y de acomodo (de uno mismo) y mantener ese objeto como diferente..." (Todorov 2007 [1989] 373).

Nos encontramos pues, a nuestro criterio, ante un claro proceso de autoexotización de la componente islámica de la cultura ibérica.

La idea de la existencia de un ente denominado "España" asociado fundamentalmente a Europa, Occidente y la Cristiandad es una construcción moderna que no da cuenta en absoluto de los procesos históricos medievales ni temprano-modernos como el ejemplo que hoy nos ocupa, es recién avanzada la Edad Moderna que la “occidentalización” se hace notoria y sus categorías adquieren validez.

3 Tal es el caso de los libros "Huellas del Islam en la literatura española. De Juan Ruiz a Juan Goytisolo" de Luce López Baralt, Hiperion, Madrid, 1985 y "La sombras del Islam en la conquista de América” de Hernán Taboada, FCE, México, 2004. Ambos excelentes estudios, que tratan de mostrar la presencia de la componente islámica en la edad Moderna pero que pese a ello no se desligan de vocablos que sostienen la idea de una referencia pasada o distante de la cual se manifiestan hoy proyecciones indirectas. Por nuestra parte, también hemos caído ocasionalmente en la misma trampa con la palabra "footprint", pero por lo general preferimos la palabra "rasgos" u otras variantes, pues entendemos refieren a una característica aún vigente y presente (ver bibliografía). 
Aun así, e incluso en el presente, la lengua en la que nos expresamos contiene un altísimo porcentaje de arabismos (la letra "a" en nuestros diccionarios ocupa un volumen muy superior a todas las demás por la presencia de palabras árabes que se castellanizaron precedidas por su artículo) lo cual significa que hasta en las expresiones, lo islámico lejos de ser una lejana influencia es un componente vivo que reside en el corazón mismo de la cultura iberoamericana.

Es decir, lo que para el periodo es regla (hibridez y vigencia de lo islámico) se explica como excepción y la excepción (casos "puros") como regla. Hasta que esta visión perversa y racista no sea sustituida, las explicaciones sobre la arquitectura del periodo serán necesariamente incompletas e inexactas.

\section{El Palacio Avellaneda, presentación de la obra}

Digamos primero que esta relectura podría hacerse sobre casi cualquier otra obra del periodo, de alguna manera eso tratamos de hacer en nuestra investigación doctoral (Martínez Nespral, 2007).

Existen numerosos ejemplos en diversos tipos de edificios ubicados a lo largo de la Península Ibérica, destacándose los grandes palacios de la nobleza como por ejemplo el de los Duques del Infantado en Guadalajara o el del Marqués de Tarifa en Sevilla, más conocido como "Casa de Pilatos".

Un común denominador en las obras de la época es la presencia de zócalos de azulejería y artesonados mudéjares que coexisten con piezas escultóricas o motivos ornamentales renacentistas.

Pasemos pues a explicar los motivos por los que nos concentrarnos en el Palacio Avellaneda.

En principio, la arquitectura palaciega es un ejemplo destacado porque, como ha sucedido en otros periodos, debido a su escala y calidad tiende a conservarse dando testimonio de su época.

Aun así, y limitándonos a los edificios construidos ex novo a fines del siglo XV y comienzos del XVI que son los que dan una muestra más acabada de este periodo de cruce, como ya hemos expresado, el Avellaneda no es naturalmente el único ejemplo, ni siquiera sería el más importante. 
Pero si es, a nuestro criterio, el que mejor y más íntegramente se ha conservado. Por el contrario, muchos se perdieron, otros tuvieron un proceso "aluvional" donde se fueron incorporando distintas arquitecturas propias de cada época y el que tal vez sería el más importante, el Palacio de los Duques del Infantado en Guadalajara, fue prácticamente destruido por la aviación de los sublevados en la Guerra Civil por lo que perdió buena parte de sus rasgos más significativos, como sus excepcionales artesonados de los cuales solo han quedado algunos testimonios fotográficos anteriores a la conflagración.

Por el contrario, el Avellaneda, "perdido" en un pequeño poblado castellano en el límite entre Burgos y Soria, si bien estuvo mucho tiempo abandonado, por una serie de hechos que podrían considerarse hasta azarosos se preservó en buena medida hasta el presente.

Vayamos a una breve presentación de la obra.

No hay una fecha exacta para el inicio de su construcción, pero sabemos que tuvo lugar en el primer tercio del siglo XVI. Se lo conoce también como Palacio de los Condes de Miranda pues fueron éstos, señores de la zona, quienes lo encomendaron.

Tampoco se puede asignar un autor específico para el edificio, aunque la historiografía entiende, sin tener certeza, que intervino el maestro Francisco de Colonia, recordado por sus trabajos en la catedral de Burgos entre otros.

De cualquier manera, y como veremos al avanzar en los detalles del edificio, si es que Francisco de Colonia en realidad tuvo actuación en este edificio, esta estuvo limitada a algunos aspectos pues varios elementos difieren sustancialmente con respecto al resto de sus obras, de claras características tardo góticas o proto renacentistas.

El sector principal tiene una configuración tradicional para este tipo de ejemplos, con un patio central rodeado por un volumen de dos plantas y cubierta de tejas.

Como es usual en estos casos, la escalera mayor se ubica en una esquina del patio y los salones principales en la planta alta cubiertos por artesonados de madera.

Su período de auge estuvo entre los siglos XVI y XVII, época en que constituía la cabeza de "los estados" o territorios en poder de sus propietarios. 
Avanzado el siglo XVII, la centralización del poder real, hizo que los nobles requirieran de estancias cada vez más prolongadas en la Corte, lugar donde todo lo importante sucedía y, con la confirmación de Madrid como capital, poco a poco las principales familias nobles se fueron asentando en nuevos palacios que construyeron en esta ciudad, retornando a sus estados en escasas oportunidades, sólo para ciertas fiestas locales o períodos de descanso.

A partir de la ausencia de sus propietarios, el palacio fue cayendo en un paulatino deterioro, acentuado por los daños ocasionados por la invasión napoleónica y la muerte sin descendencia de la última representante de la familia que lo construyó.

Como parte de la sucesión, el edificio termina incorporándose al vasto patrimonio de los Duques de Alba con lo que se produce un virtual abandono del mismo.

Su oportuna incorporación como bien protegido en 1923 impidió que el norteamericano Arthur Byne, celebre estudioso y expoliador de bienes culturales españoles, lo comprara para desarmarlo y venderlo al magnate William Randolph Hearst en 1925 quien pensaba usar las partes para la construcción de su célebre "castillo" californiano.

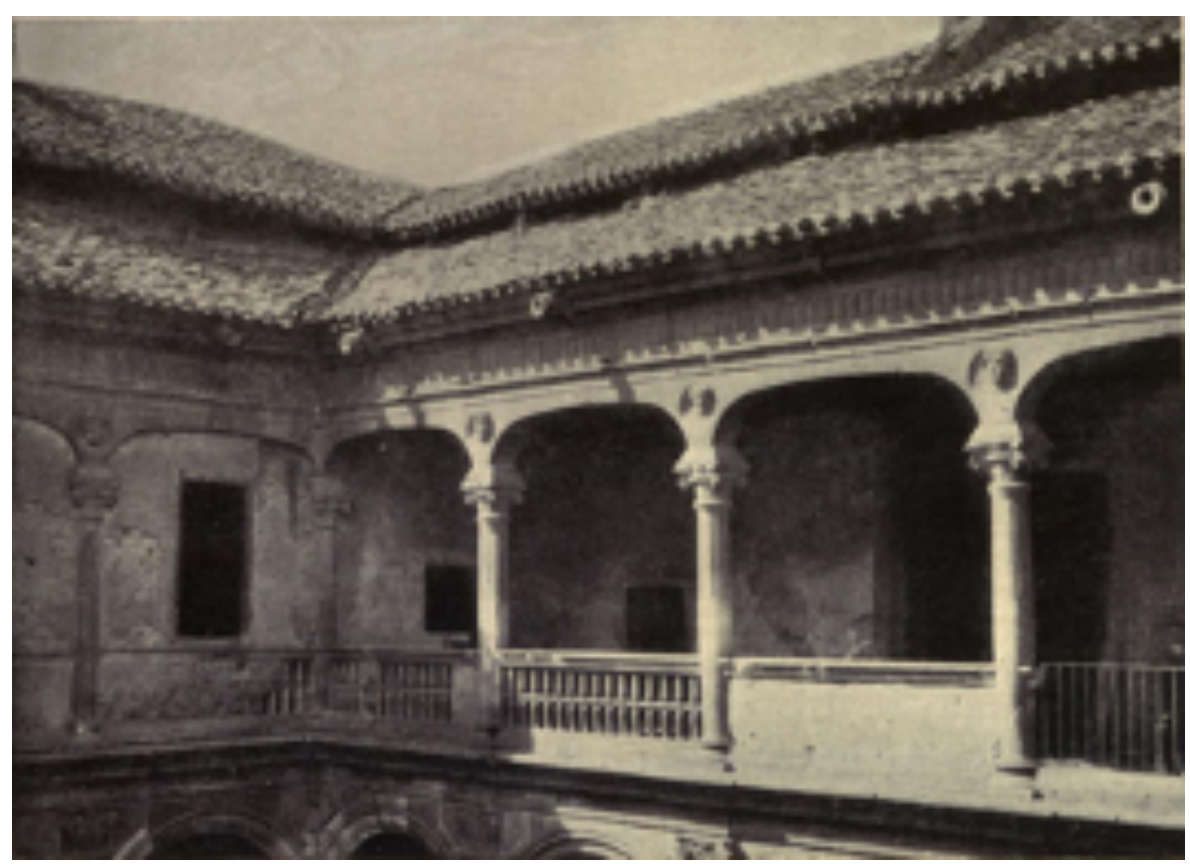




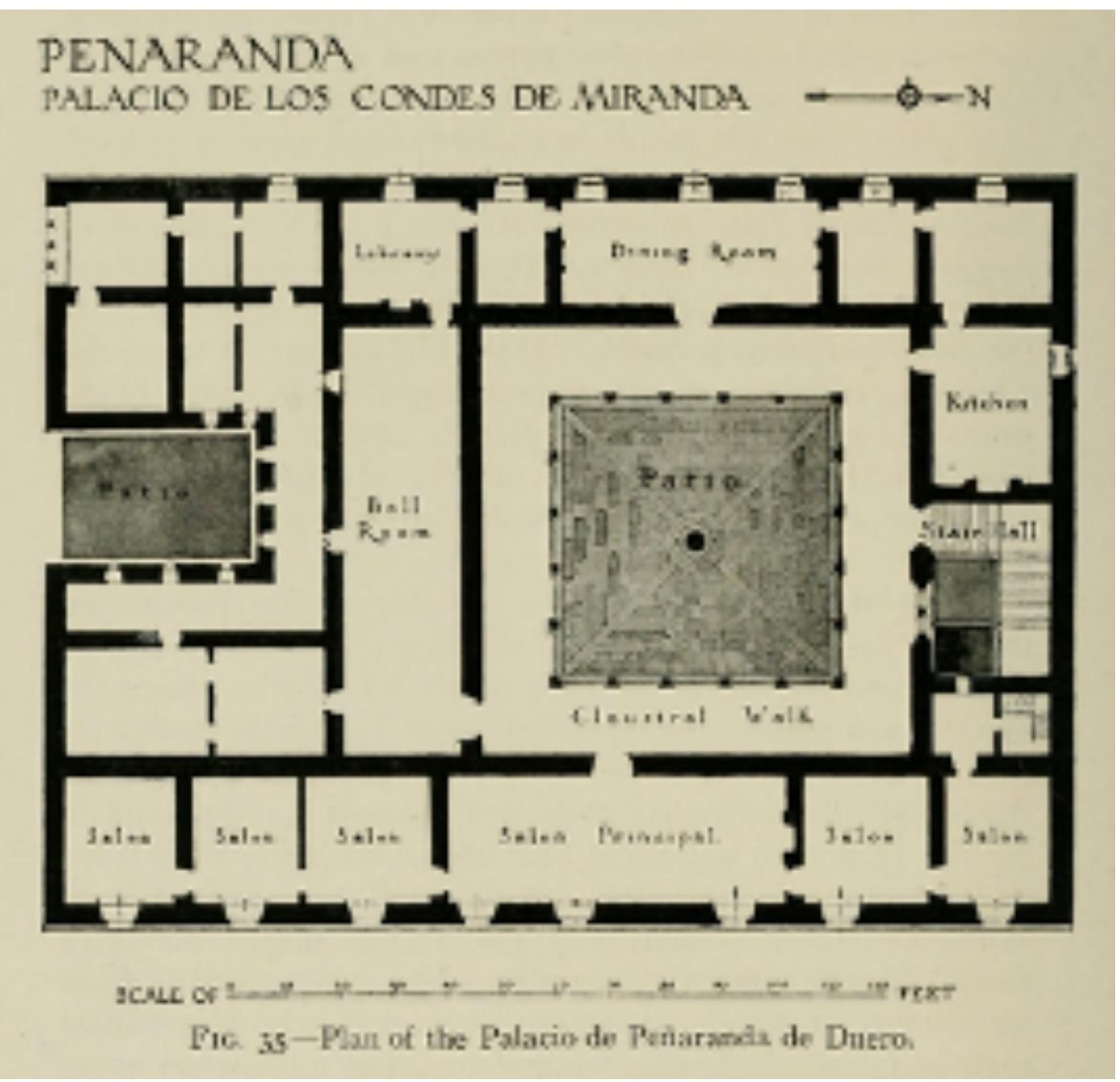

Figuras 1 y 2: Patio del Palacio de los Condes de Miranda en Peñaranda de Duero hacia el año 1916 y planta noble del mismo edificio. Publicadas en el libro: "Spanish architecture of the sixteenth century; general view of the plateresque and Herrera styles" (1917) de Arthur Byne.

Luego de la Guerra Civil, el palacio pasa a manos del gobierno del dictador Franco que restaura la parte principal y en un bloque anexo se instala una escuela para altos mandos de la falange.

Esta restauración preservó y consolidó los artesonados de madera y la estructura general del edificio, pero se perdieron la policromía de los artesonados y revestimientos de azulejería.

Actualmente es propiedad de la Junta de Castilla y León, que ha hecho otras intervenciones en sectores nuevos y restauraciones del área principal. Como en varios monumentos "de provincia" se puede ingresar en ciertos horarios con un guía de la oficina de turismo local, pero, alejado de los grandes centros urbanos y de los principales atractivos turísticos, es escasamente visitado.

Por otra parte, la obra ha tenido un limitado interés para la historiografía, sólo le han dedicado artículos, o ha sido nombrada en textos generales, 
a comienzos del siglo XX, como el libro ya mencionado de Byne (1917) y algunas obras de Vicente Lamperez y Romea (1912-1922).

Más recientemente el principal aporte es un artículo de Eduardo Carazo (1997) que fundamentalmente hace una completa descripción e historia del edificio, pero no avanza demasiado en cuestiones interpretativas y, por el contrario, continúa con las categorías anteriores, definiendo a la obra como parte de “...nuestra arquitectura civil del primer Renacimiento..." (Carazo, 1997, p. 508).

Existe también otro texto reciente, un artículo de Jorge Martínez Montero (2005), aunque específicamente dedicado a la escalera, y refiriéndola también a los modelos renacentistas.

Por el contrario, el Palacio, como la mayor parte de la arquitectura del período, se podría entender a partir de la utilización de soluciones islámico-mediterráneas en lo estructural y la combinación de estas con las influencias renacentistas italianas en determinados elementos decorativos.

Así, en lo tipológico y compositivo se trata de un edificio de patio con un esquema visual y circulatorio a través de acodamientos para garantizar la privacidad, un recurso típico de la arquitectura islámica (aquello que el célebre historiador de la arquitectura española, Fernando Chueca Goitía denominó erróneamente “ejes quebrados”) Así, la puerta principal da acceso a un zaguán y la comunicación de éste con el patio -y de allí con el resto del edificio- se realiza a través de una arcada notoriamente desplazada del eje de la puerta, por lo cual fuerza el giro a noventa grados (acodamiento) e impide las visuales desde la calle hacia el patio.

Por otra parte, y en cuanto a los materiales y técnicas constructivas empleadas, la estructura se resuelve con una caja muraria que se reviste en su superficie con zócalos de cerámica esmaltada en la parte inferior de los paramentos, yesería en la superior mientras que las cubiertas de las diferentes crujías se resuelven con artesonados de madera y tejas árabes.

Esta descripción, en lo atinente a los criterios de diseño arquitectónico, sería válida para la Alhambra, los alcázares de Sevilla, Segovia o Madrid entre tantos otros edificios construidos indistintamente para musulmanes o cristianos. Las diferencias entre ellos no están en la estructura de su composición sino en elementos decorativos propios de cada período, aunque todos ellos compartieron la conjunción de elementos islámicos, góticos y renacentistas. 


\section{Más es más, una relectura "Venturiana"}

Recientemente tuvimos oportunidad de "descubrir" el palacio Avellaneda, primero de forma casual en 2018 y luego con un segundo viaje organizado especialmente para visitarlo en 2019, y más allá de la maravilla ante una obra sin duda "...tan importante como desconocida..." (Carazo, 1997, p. 508), lo que fundamentalmente nos sorprendió fue la expresa voluntad de sus anónimos autores de presentar de forma paralela y "balanceada" elementos de origen islámico y renacentistas.

Esta deliberada combinación se ve en cada detalle, el más llamativo de ellos es la tradicional yesería islámica con la que se resuelve la decoración de los muros pero que en este caso alterna sistemáticamente cual un código binario motivos renacentistas "intercalados" con los islámicos, materializados ambos con la misma técnica constructiva.

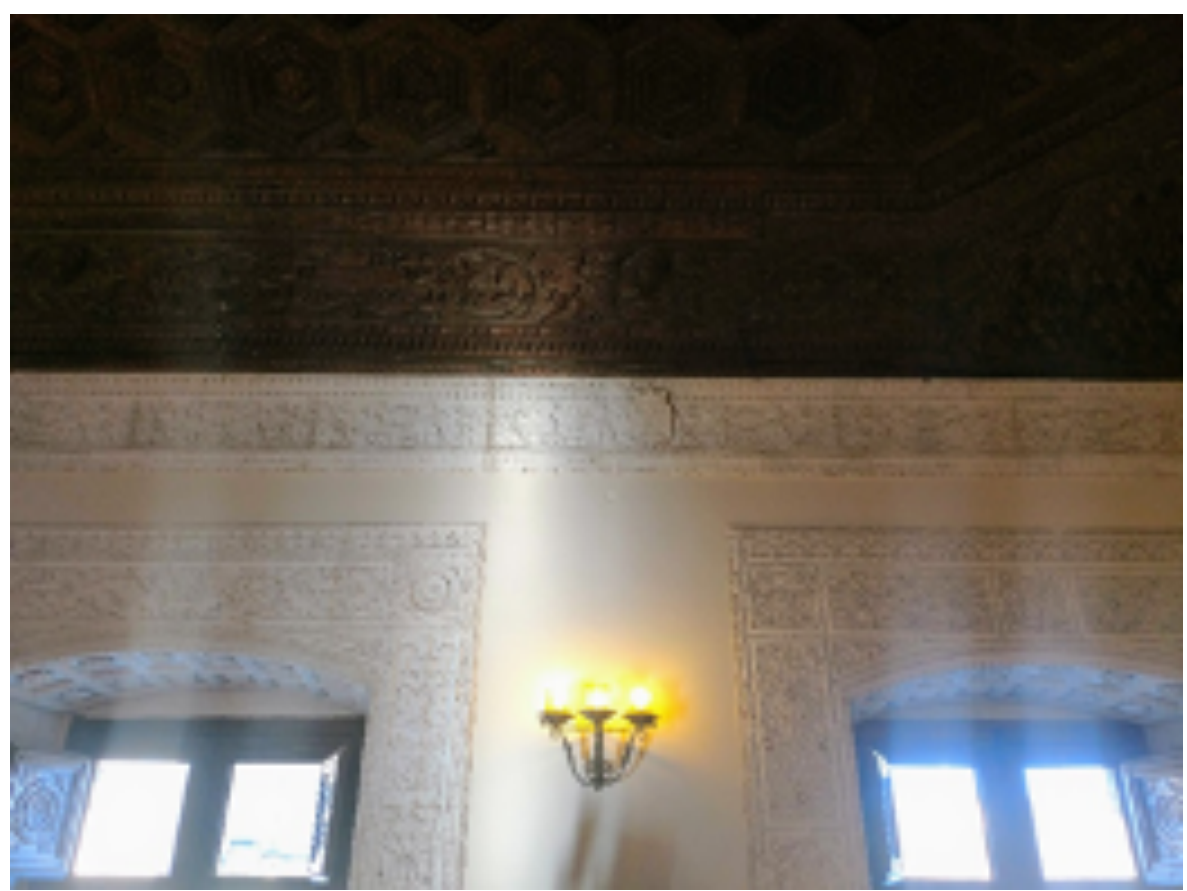

Figura 3: Yesería alternada con motivos islámicos y renacentistas en el Palacio Avellaneda. Fotografía del autor.

Pero del mismo modo los artesonados combinan módulos hexagonales propios del renacimiento con mocárabes y la escalera incluye una balaustrada y columnas de mármol a la manera italiana, deliberadamente yuxtapuestas con una cenefa de yesería y artesonados islámicos.

Es constante en el edificio esta exhibición de un simultáneo dominio de ambos códigos estéticos, tanto en lo que refiere a la capacidad técnica de sus 
autores para generar indistintamente soluciones islámicas o renacentistas como en la capacidad de sus comitentes de habitar y valorar un edificio definido de forma equivalente a través de ambas estéticas.

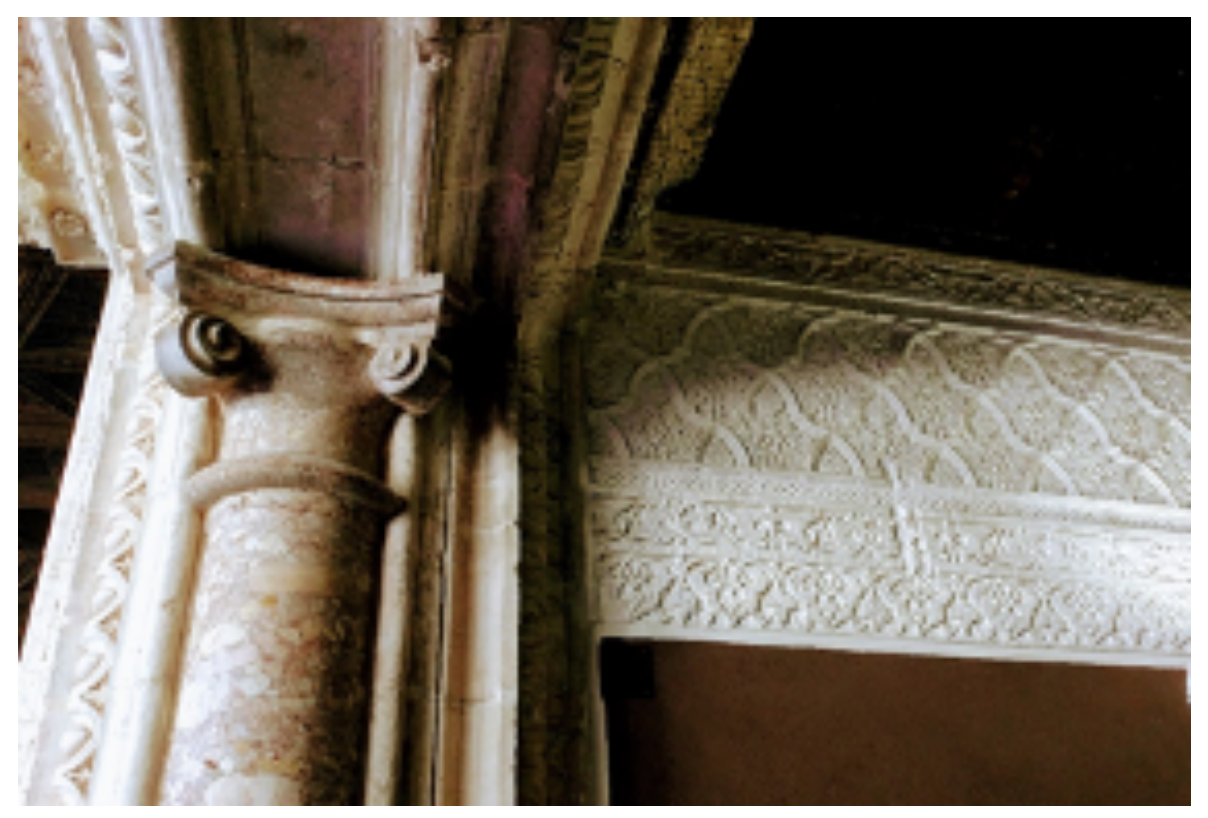

Figura 4: Combinación de yesería islámica y órdenes renacentistas en el Palacio Avellaneda. Fotografía del autor.

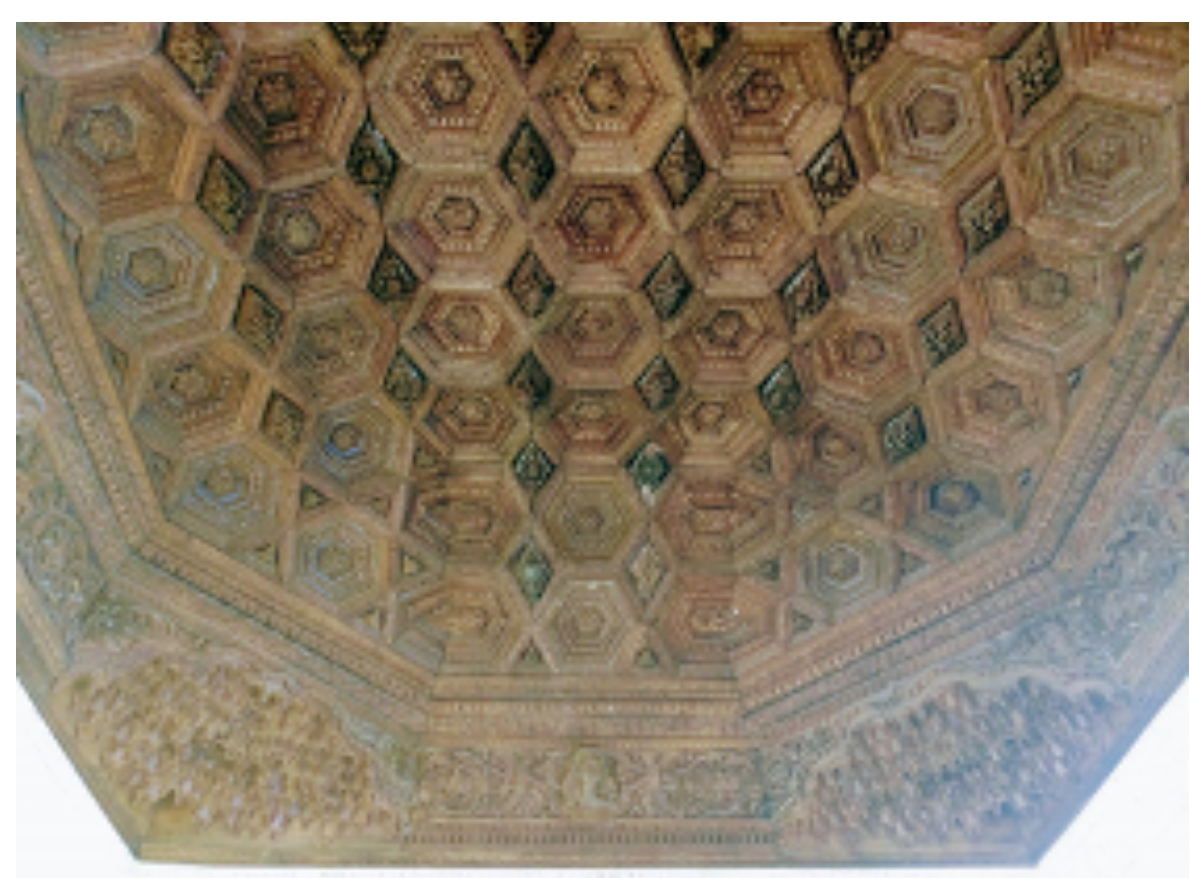

Figura 5: Artesonado con módulos hexagonales renacentistas y mocárabes islámicos en el Palacio Avellaneda. Fotografía del autor. 
El Avellaneda todo es, de alguna manera, un edificio "bilingüe" donde lo hispano-árabe está permanentemente imbricado con lo ítalo-latino, como sucede en nuestro idioma.

Aquello que en el texto de Carazo se define como "...continuas discrepancias entre los distintos procedimientos decorativos y compositivos..." (Carazo, 1997, p. 529) a nuestro criterio se puede explicar como el reflejo de una sociedad que no puede ser entendida en términos de "esto o aquello" sino que por el contrario requiere indispensablemente de la idea de "esto $y$ aquello".

Es, por lo tanto, esta complejidad y las consecuentes contradicciones que surgen al querer interpretar la arquitectura española en general, y en particular la de este período, desde modelos puristas (que inevitablemente tienen una conexión con las ideas del racismo) las que nos hacen ligar a este tipo de arquitecturas con el posmodernismo.

El palacio en su conjunto es una demostración palpable de la idea Venturiana de "menos es aburrido", no sólo por la complejidad ornamental de todas sus superficies, sino, y fundamentalmente por su bilingüismo conceptual. Como en las obras de Robert Venturi que no quieren renunciar ni a los esquemas modernos ni a las formas tradicionales.

Vista desde Argentina, el Palacio Avellaneda es sin duda una obra distante en tiempo y espacio, y, la voluntad de ligarla con la narrativa Venturiana, otro fenómeno también distante y en apariencia distinto e inconexo con el edificio en cuestión, podría ser perfectamente interpretada como un tour de force.

Pero creemos con Todorov que "La calidad de la observación depende de la posición del observador.” (Todorov (2007 [1989]) 403) por ello entendemos que la visión de España desde Argentina que hemos tratado de construir a lo largo de nuestra carrera académica en las últimas décadas es posible justamente pues se realiza desde Argentina, lejos de los límites que imponen a los nativos los procesos de construcción de la identidad española. No significa que desde aquí veamos mejor o peor, sino que, desde aquí, necesariamente, vemos distinto.

Por otra parte, una respuesta a nuestro interés por la obra puede surgir de la explicación de Todorov sobre la alegoría: "La alegoría dice una cosa, y da a entender otra; el alegorista habla de un pueblo (extranjero) para debatir sobre una cosa distinta a ese pueblo - sobre un problema que concierne al mismo alegorista y a su propia cultura" (Todorov (2007 [1989]) 393). 
Seguramente nuestra atracción por el Palacio Avellaneda en particular (y por la compleja arquitectura peninsular en general) a la vez que por la lógica arquitectónica Venturiana surgen como una forma de reacción a problemas mucho más cercanos, como la idealización de las estéticas eurocéntricas del movimiento moderno y su lectura simplista a lo "menos es más” de las que, estamos convencidos, la enseñanza de la arquitectura en Argentina sigue siendo víctima.

\section{Epílogo}

Robert Venturi falleció el 18 de septiembre de 2018, su obra escrita y construida y particularmente "Complexity and contradiction in contemporary architecture”, a mi criterio el mejor libro de arquitectura del siglo XX, renovaron esta disciplina hasta entonces prisionera de una propuesta mesiánica y colonial que se presentaba como la única alternativa.

Sirva este texto como modesto homenaje y muestra de gratitud de uno de los tantos que fuimos transformados por su obra. 


\section{Q Bibliografía}

»Byne, A. (1917). Spanish architecture of the sixteenth century; general view of the plateresque and Herrera styles. Nueva York: G. P. Putnam's Sons.

»Carazo, E. (1997). El Palacio de los Condes de Miranda en Peñaranda de Duero. Boletín de la Academia de Bellas Artes de San Fernando, 87, 505544.

»Castillo Oreja, M. A. (1985). La proyección del arte islámico en la arquitectura de nuestro primer renacimiento: el estilo Cisneros. Anales del Instituto de estudios madrileños, 22, 55-63. Madrid: C.S.I.C.

» Castro, A. (1948). España en su historia. Cristianos moros y judíos. Buenos Aires: Losada.

» de los Ríos, J. (1872). El estilo mudéjar en la arquitectura. Madrid: Imprenta de Manuel Tello.

"Fox, I. (1997). La invención de España. Nacionalismo liberal e identidad nacional. Madrid: Cátedra.

»González Ferrín, E. (2018). Cuando fuimos árabes. Córdoba: Almuzara.

»Kamen, H. (2020). La invención de España. Madrid: Espasa.

» Lampérez y Romea, V. (1922) Arquitectura Civil Española de los siglos I al XVIII. Madrid: Saturnino Calleja.

»Lampérez y Romea, V. (1912). El Palacio de los Condes de Miranda en Peñaranda de Duero. Boletín de la Sociedad Española de Excursiones, XX, 146-151.

» López Baralt, L. (1985). Huellas del Islam en la literatura española. De Juan Ruiz a Juan Goytisolo. Madrid: Hiperion.

" Martínez Montero, J. (2005). La escalera del Palacio de los Condes de Miranda en Peñaranda de Duero, Burgos. De Arte, Revista de Historia del Arte, 4, 75-87.

" Martínez Nespral, F. (2007). Un juego de espejos. Rasgos mudéjares de la arquitectura y el habitar en la España de los Austrias (siglos XVI-XVII). Buenos Aires: Nobuko.

»Martínez Nespral, F. (2017). Islamic footprints in Latin American Architecture. Ponencia inédita presentada al Congreso Annual de la College art Association, Nueva York.

" Martínez Nespral, F. (2018). Architectural links between the Islamic world and Latin America. Módulo docente realizado para la red Global Architectural History Teaching Collaborative, disponible on-line en www.gahtc.org

" Martínez Nespral, F. (2020). Nuestro cercano Oriente. Rasgos Islámicos de la arquitectura y la ciudad en Latinoamérica. En: Loredo, R. y Lara, F., Apuntes sobre decolonización, arquitectura y ciudad en las Américas México, Colofón (en prensa).

» Ortega y Gasset, J. (1921). España invertebrada. Madrid: Espasa Calpe. 
» Taboada, H. (2004). La sombras del Islam en la conquista de América. México: Siglo XXI.

"Todorov, T. (2007 [1989]). Nosotros y los otros. México: Siglo XXI.

»Venturi, R. (1968 [1966]). Complexity and contradiction in contemporary architecture. Nueva York: The Museum of Modern Art papers on Architecture. 